\title{
Searches for charged Higgs bosons, supersymmetry, and exotica with tau leptons with the ATLAS and CMS detectors at the LHC
}

\author{
Ryan Reece on behalf of the ATLAS and CMS collaborations
}

ryan.reece@cern.ch

\begin{abstract}
Results are presented from the ATLAS and CMS searches for charged Higgs bosons, supersymmetry, and exotica with tau leptons in the final state. The analyses discussed use a dataset corresponding to $5 \mathrm{fb}^{-1}$ of integrated luminosity from $\sqrt{s}=7 \mathrm{TeV}$ proton-proton collisions at the LHC in 2011 .
\end{abstract}

Keywords: ATLAS, CMS, tau, charged Higgs, supersymmetry, $Z^{\prime}$, leptoquarks

\section{Introduction}

Hadronic tau decays play an important role in searches for new physics at the LHC [1] because tau leptons often have preferred couplings to new physics. This talk reviews recent results from ATLAS [2] and CMS [3] searches for charged Higgs bosons, supersymmetry, and exotica with hadronic tau decays, using $5 \mathrm{fb}^{-1}$ of integrated luminosity from $\sqrt{s}=7 \mathrm{TeV}$ proton-proton collisions during the year 2011. Both ATLAS [4] and CMS [5] have sophisticated multivariate techniques for identifying hadronic tau decays by selecting well-isolated calorimeter clusters closely associated to one or three tracks.

\section{Searches for charged Higgs bosons}

A general class of theories beyond the Standard Model (SM) involve two Higgs doublets of complex scalar fields instead of the single Higgs doublet of the SM. The two Higgs doublet model (2HDM) is the defining characteristic of the Minimal Supersymmetric Standard Model (MSSM). In 2HDMs, electroweak symmetry breaking results in five spin- 0 Higgs bosons, three that are neutral: $h^{0}, H^{0}, A^{0}$, and two that have electromagnetic charge: $H^{ \pm}$. The parameter $\tan \beta$ is defined as the ratio of vacuum expectation values of the Higgs doublets, and has a large effect on the phenomenology of 2HDMs. For $\tan \beta \gtrsim 2$, charged Higgs bosons decay dominantly to tau leptons through $H^{ \pm} \rightarrow \tau^{ \pm} v$, making tau final states important for searching for charged Higgs bosons. If the mass of the charged Higgs boson is less than the mass of the top quark, then charged Higgs bosons are produced at hadron colliders dominantly in $t \bar{t}$ events, where the $H^{ \pm}$takes the place of an emitted $W^{ \pm}$ when the top decays promptly to a bottom quark. Therefore searches for charged Higgs bosons involve looking for an excess of tau final states in $t \bar{t}$ events.

ATLAS [6] combines three channels depending on the decay products in its search for charged Higgs bosons: $b b(q q)\left(\tau_{\mathrm{had}} v\right), b b(\ell v)\left(\tau_{\mathrm{had}} v\right)$, and $b b(q q)(\ell v)$, where $\tau_{\text {had }}$ denotes the reconstructed visible part of a hadronic tau decay. In the most sensitive channel, $b b(q q)\left(\tau_{\text {had }} v\right)$, events are triggered by a $\tau_{\text {had }}$ with $p_{\mathrm{T}}>29 \mathrm{GeV}$ and missing transverse momentum $\left(E_{\mathrm{T}}^{\mathrm{miss}}\right)$ $>35 \mathrm{GeV}$. Offline, events are selected which have 4 jets with $p_{\mathrm{T}}>20 \mathrm{GeV}$, of which at least one must be tagged as a $b$-jet, exactly one $\tau_{\text {had }}$ with $p_{\mathrm{T}}>40 \mathrm{GeV}$, no electrons or muons, and $E_{\mathrm{T}}^{\text {miss }}>65 \mathrm{GeV}$. The three-jet combination with the highest $p_{\mathrm{T}}^{j j b}$ must have $m_{j j b} \in[120,140] \mathrm{GeV}$, being consistent with a top quark decay. To further suppress background multijet events with fake $E_{\mathrm{T}}^{\mathrm{miss}}$, the $E_{\mathrm{T}}^{\mathrm{miss}}$ significance defined 
as $E_{\mathrm{T}}^{\mathrm{miss}} /\left(0.5 \mathrm{GeV}^{1 / 2} \cdot \sqrt{\sum p_{\mathrm{T}}}\right)$ is required to be larger than 13, where $\sum p_{\mathrm{T}}$ denotes the sum of the $p_{\mathrm{T}}$ of all tracks associated with the primary vertex.

Figure 1 shows the transverse mass of the combination of the $\tau_{\text {had }}$ and the $E_{\mathrm{T}}^{\text {miss }}$, which is used to calculate a profile likelihood ratio for testing the compatibility of the signal hypothesis. No significant deviation from the SM expectation is observed and 95\% CL limits are set using the frequentist CLs technique in the $\tan \beta$ vs. $m_{H^{+}}$plane assuming the $m_{h}^{\max }$ scenario of the MSSM, as shown in Figure 2. CMS has a similar result [7], both excluding charged Higgs bosons with masses $\lesssim 150 \mathrm{GeV}$ for $\tan \beta \gtrsim 15$.

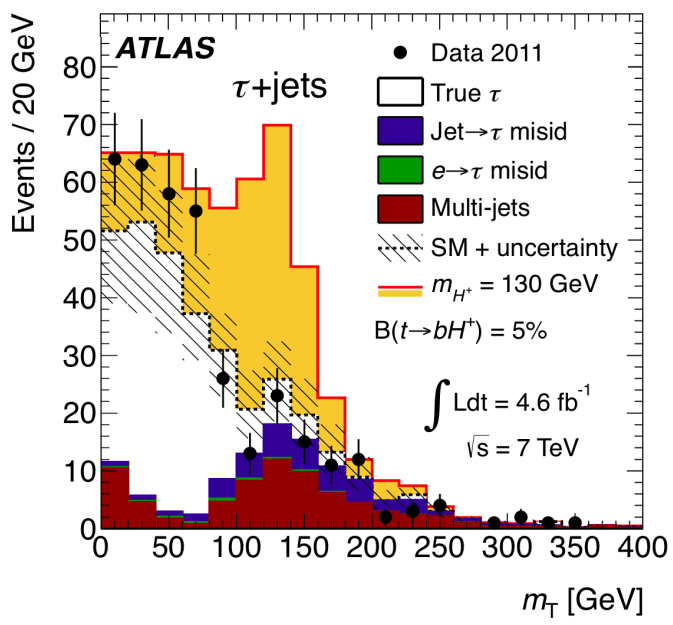

Figure 1: Distribution of $m_{\mathrm{T}}$ after all event selection in the $\tau_{\text {had }}+$ jets channel. The solid line shows the predicted contribution of signal+background in the presence of a charged Higgs boson with $m_{\mathrm{H}+}=$ $130 \mathrm{GeV}$, assuming $\mathrm{BR}\left(t \rightarrow b H^{+}\right)=5 \%$ and $\mathrm{BR}\left(H^{+} \rightarrow \tau v\right)=$ $100 \%[6]$.

\section{Searches for SUSY in multilepton events with jets and $E_{\mathrm{T}}^{\text {miss }}$}

Supersymmetry (SUSY) is a natural extension of the Standard Model that introduces a symmetry relating fermions and bosons. In R-parity conserving models, there is a Lightest Supersymmetric Particle (LSP) that must be stable to conserve the R-partiy quantum number. The LSP is presumably only weakly interacting. It therefore goes undetected and results in events with significant $E_{\mathrm{T}}^{\text {miss }}$ in addition to the jets and/or leptons produced in the cascade of a SUSY decay. Both CMS and ATLAS perform searches for SUSY in events with hadronic tau decays in the final state.

In one of the searches preformed by CMS [8], events are selected with same-sign leptons. All combinations

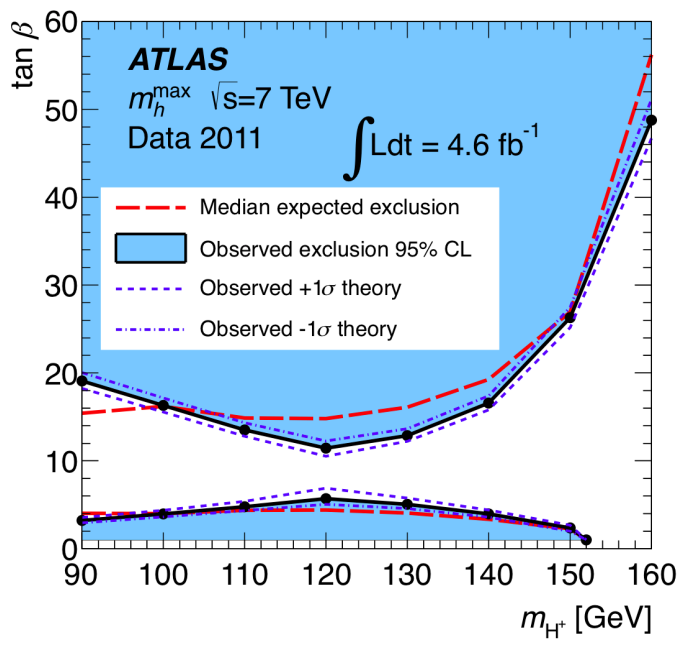

Figure 2: Combined 95\% CL exclusion limits on $\tan \beta$ as a function of $m_{\mathrm{H}^{+}}$in the context of the MSSM $m_{h}^{\max }$ scenario [6].

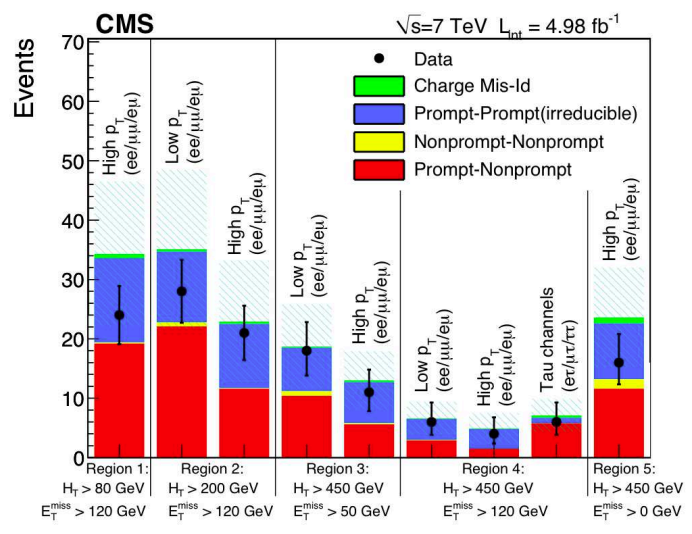

Figure 3: Summary of background predictions and observed yields in the various search regions. The hatched bands represent the total uncertainty on the background predictions. [8].

of charged leptons are considered: $e e, \mu \mu, \tau_{\text {had }} \tau_{\text {had }}$, $e \mu, e \tau_{\text {had }}$, and $\mu \tau_{\text {had }}$ are considered. The search targets R-partiy conserving SUSY, and therefore significant $E_{\mathrm{T}}^{\text {miss }}$ and $H_{\mathrm{T}}$ are expected, where $H_{\mathrm{T}}$ is defined as the scalar sum of the $p_{\mathrm{T}}$ of all selected jets in the event. Since the mass differences of hypothetical SUSY particles can strongly affect the event kinematics, five different signal regions are defined depending on the $E_{\mathrm{T}}^{\text {miss }}$ and $H_{\mathrm{T}}$ thresholds. Figure 3 shows the summary of the predicted and observed events in each signal region. No significant excess is observed and exclusion limits are set in the universal mass parameter plane of the Constrained Minimal Supersymmetric Standard Model (CMSSM) as shown in Figure 4, significantly extending the previous exclusions from LEP. A similar result 


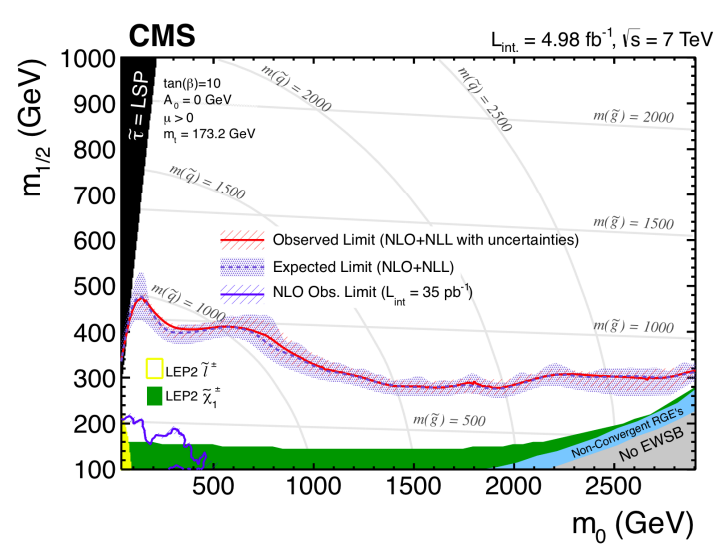

Figure 4: The region below the red curve is excluded in at $95 \% \mathrm{CL}$ in the context of the CMSSM. The hatched region corresponds to the theoretical uncertainties on the cross section, whereas the shaded region shows the experimental errors. Previous exclusions from LEP are also shown [8].

is also derived in an analysis of events with oppositesign leptons [9].

CMS also performs an analysis of multilepton events [10], categorizing all events with three or four leptons of each flavor: $e, \mu$, or $\tau_{\text {had }}$. Events are categorized by the number of electrons or muons, number of hadronic tau decays, $E_{\mathrm{T}}^{\text {miss }}$ threshold, $H_{\mathrm{T}}$ threshold, $S_{\mathrm{T}}$ threshold, and with or without an opposite-sign same-flavor lepton pair, for a total of 106 signal regions. $S_{\mathrm{T}}$ denotes the scalar sum of all selected jets $\left(H_{\mathrm{T}}\right)$ as well as the selected leptons and $E_{\mathrm{T}}^{\text {miss }}$. No significant excess is observed, and limits are set in the squarkgluino mass plane assuming R-partiy violating (RPV) couplings. Figure 5 shows the exclusion assuming RPV coupling $\lambda_{e \mu \tau}$, which would produce three-lepton events with an electron, muon, and tau from the cascade of a SUSY decay.

ATLAS performs a search for supersymmetric decays with taus, jets, and $E_{\mathrm{T}}^{\mathrm{miss}}$ in the final state [11]. Four channels are combined in the search, including events with exactly one $\tau_{\text {had }}$ and no leptons, two $\tau_{\text {had }}$ and no leptons, one electron and one $\tau_{\text {had }}$, and one muon and one $\tau_{\text {had }}$. In the $\mu+\tau_{\text {had }}$ channel, for example, the SM background is dominated by $W+$ jet and $t \bar{t}$ events. The backgrounds are suppressed by requiring the transverse mass between the muon and $E_{\mathrm{T}}^{\text {miss }}$ to be greater than $100 \mathrm{GeV}$, and by requiring that the effective mass, $m_{\mathrm{eff}}$, defined as the scalar sum of the $p_{\mathrm{T}}$ of the $\mu, \tau_{\text {had }}$, selected jets, and $E_{\mathrm{T}}^{\text {miss }}$, to be greater than $1000 \mathrm{GeV}$. Figure 6 shows the $m_{\mathrm{eff}}$ of the $\mu+\tau_{\text {had }}$ channel including all event selections except the cut on $m_{\mathrm{eff}}$. No significant excess is observed, and 95\% CL limits are set using

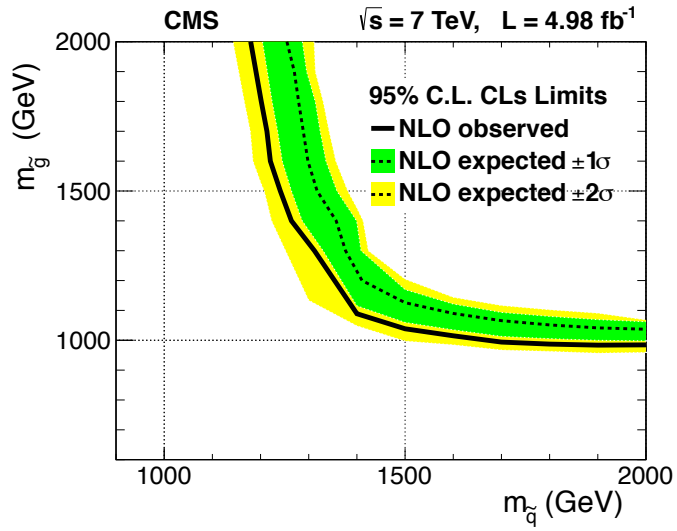

Figure 5: The region on the left of the curve is excluded at the 95\% $\mathrm{CL}$ in the squark-gluino mass plane for RPV coupling $\lambda_{e \mu \tau}[10]$.

the CLs technique in the $\tan \beta$ vs $\Lambda$ plane, where $\Lambda$ is the SUSY breaking scale assuming a model with Gauge Mediated Supersymmetry Breaking (GMSB), as shown in Figure 7.

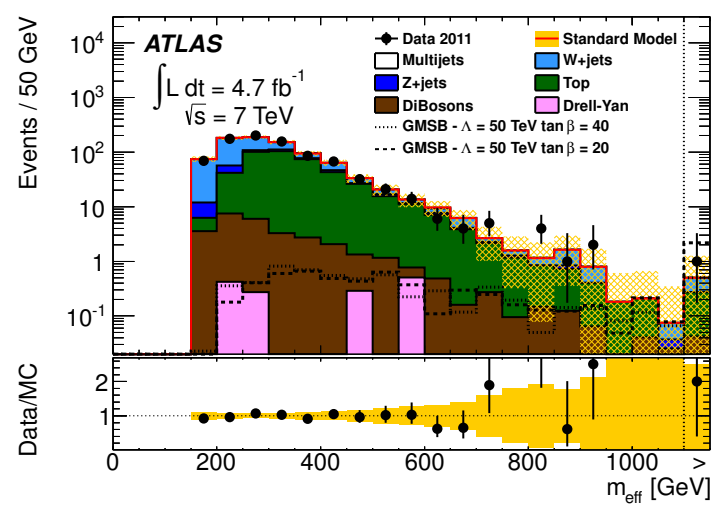

Figure 6: The effective mass distribution, $m_{\mathrm{eff}}$, of the $\mu+\tau_{\text {had }}$ channel, with all event selections except the cut on $m_{\mathrm{eff}}[11]$.

\section{Third generation leptoquark search}

Leptoquarks are hypothetical bosons predicted in many Grand Unified Theories (GUTs) and carry color, lepton, and baryon quantum numbers. The dominant production mechanisms for leptoquarks at hadron colliders are gluon-gluon fusion and quark-antiquark annihilation. Third generation leptoquarks are expected to decay predominantly to third generation SM particles: a $b$-quark and a $\tau$-lepton.

CMS searches for pairs of leptoquarks where one of the $\tau$-leptons decays leptonically and the other hadronically [12]. Events with a combination of an electron or 


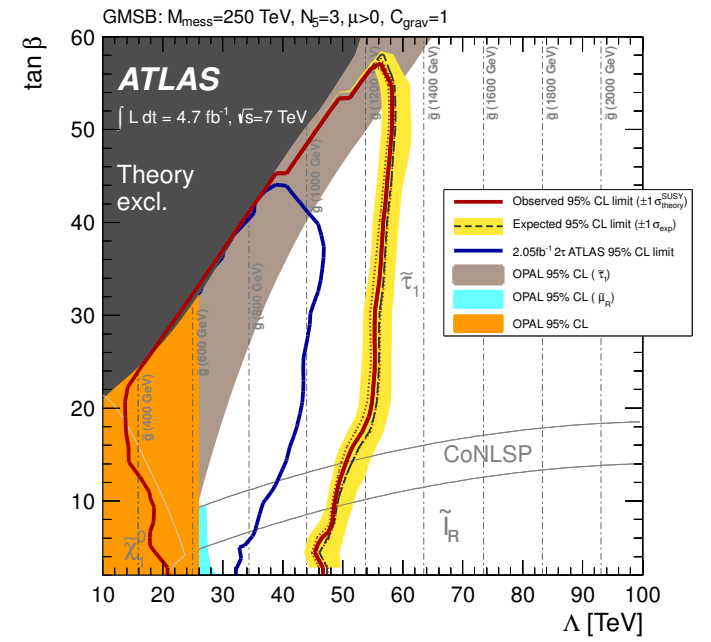

Figure 7: Expected and observed 95\% CL lower limits on the minimal GMSB model parameters $\Lambda$ and $\tan \beta$. The dark grey area indicates the region which is theoretically excluded due to unphysical sparticle mass values [11]

muon and a candidate hadronic tau decay are triggered. Offline, events are selected which have an electron or muon with $p_{\mathrm{T}}>30 \mathrm{GeV}$, a $\tau_{\text {had }}$ with opposite-signed charge and $p_{\mathrm{T}}>50 \mathrm{GeV}$, and have at least two $b$-tagged jets with $p_{\mathrm{T}}>30 \mathrm{GeV}$. The resulting event sample is dominated by $t \bar{t}$ events. To discriminate between signal and background, the mass of the $\tau_{\text {had }}$ and $b$-jet, $M_{\tau, b}$, is required to be greater than $170 \mathrm{GeV}(190 \mathrm{GeV})$ for signal hypotheses with masses up to $450 \mathrm{GeV}$ (greater than $450 \mathrm{GeV}$ ). Of the two possible pairings of the $\tau_{\text {had }}$ and $b$-jets, the one for which the mass is closest to the mass of the lepton and the other $b$-jet is chosen. No significant excess is observed, and the scalar sum of the $p_{\mathrm{T}}$ of the lepton, $\tau_{\text {had }}$, and $b$-jets, denoted $S_{\mathrm{T}}$ (shown in Figure 8), is used to set $95 \%$ CL limits using the CLs technique, as shown in Figure 9.

\section{5. $Z^{\prime} \rightarrow \tau \tau$ searches}

Many GUTs predict additional U(1) gauge symmetries which result in new gauge bosons, often denoted $Z^{\prime}$. In the Sequential Standard Model (SSM), a common benchmark, the $Z^{\prime}$ has the same couplings as the $\mathrm{SM} Z$ boson. The best limits on $Z^{\prime} \rightarrow e e / \mu \mu$ come from CMS [13] and ATLAS [14], being $m_{Z^{\prime} \text { ssm }}>2.3 \mathrm{TeV}$ and $2.2 \mathrm{TeV}$ respectively. It is important to independently test for new physics in the ditau final state because many theories predict non-universal couplings.

ATLAS [15] combines searches for $Z^{\prime} \rightarrow \tau \tau$, where both taus decay hadronically, one tau decays leptoni-

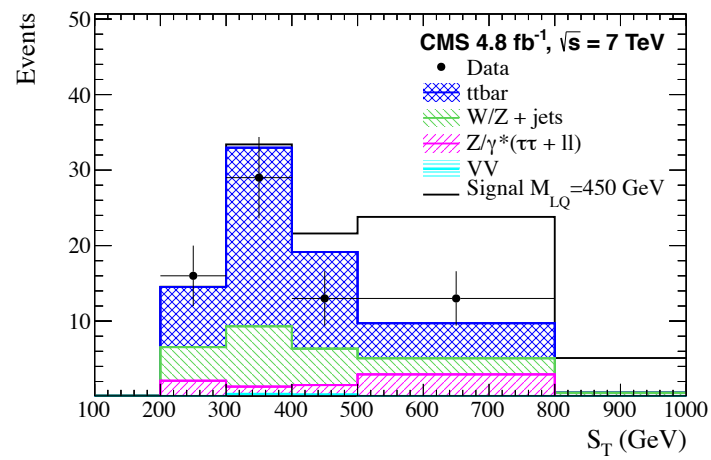

Figure 8: $S_{\mathrm{T}}$ distribution of data (black dots) and the stacked SM backgrounds (colors) and LQ signal (black line) after requiring $M_{\tau, \mathrm{b}}>$ $170 \mathrm{GeV}[12]$.

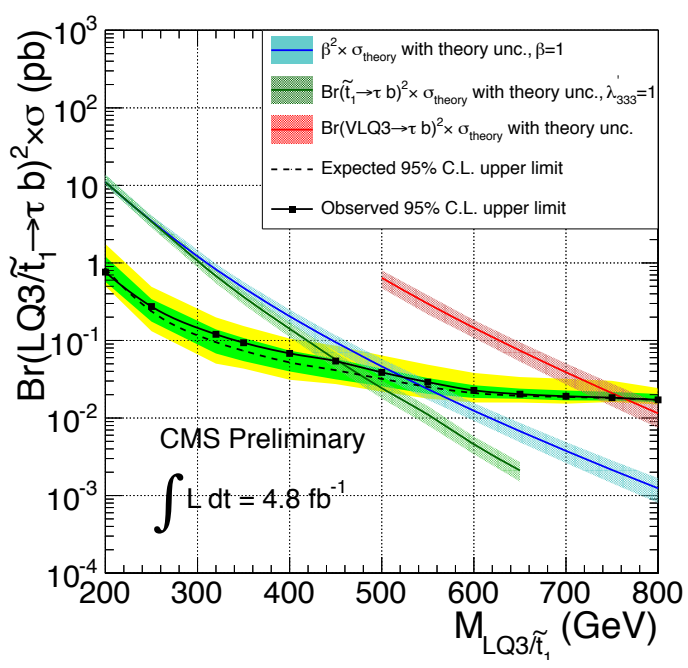

Figure 9: Expected and observed upper limit at 95\% CL on the LQ $\left(\tilde{t}_{1}\right)$ pair production cross section times $\mathrm{BR}\left(\mathrm{LQ} / \tilde{t}_{1} \rightarrow \tau b\right)$ as a function of LQ $\left(\tilde{t}_{1}\right)$ mass [12].

cally and the other hadronically, and where both taus decay leptonically with an $e \mu$ final state. The doubly hadronic channel, having the largest braching fraction, is the most sensitive. In that channel, events are triggered by either a ditau trigger with $p_{\mathrm{T}}$ thresholds of 20 and $29 \mathrm{GeV}$, or a single-tau trigger with $p_{\mathrm{T}}>125 \mathrm{GeV}$. Offline, events are selected which have two loosely identified hadronic tau decays with $p_{\mathrm{T}}>50 \mathrm{GeV}$ that have opposite-signed charges, and $\Delta \phi>2.7$, being the angle between the tau decays in the transverse plane. The total transverse mass of the combination of the two tau decays and the $E_{\mathrm{T}}^{\text {miss }}$ is calculated, shown in Figure 10, and high-mass signal regions are optimized as a function of the mass of the $Z^{\prime}$ signal. Bayesian credibility intervals are determined leading to $95 \%$ CL upper 
limits on the cross section times branching fraction for a high-mass resonance decaying into a $\tau^{+} \tau^{-}$pair as a function of the resonance mass, as shown in Figure 11. As a result, SSM $Z^{\prime}$ bosons are excluded with masses less than $1.3 \mathrm{TeV}$. The result has been updated since this conference to exclude up to $1.4 \mathrm{TeV}$ [16]. CMS performs a similar search, also excluding SSM $Z^{\prime}$ bosons decaying to $\tau^{+} \tau^{-}$with masses less than $1.4 \mathrm{TeV}$ [17].

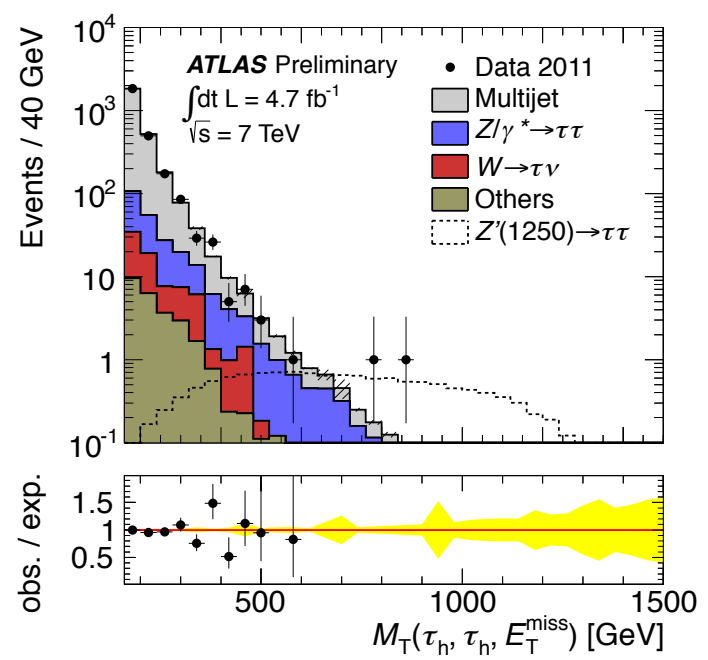

Figure 10: The distribution of the total transverse mass of the $\tau_{\text {had }}+$ $\tau_{\text {had }}+E_{\mathrm{T}}^{\text {miss }}$ combination, $M_{\mathrm{T}}$, after all event selections except the $M_{\mathrm{T}}$ threshold. [15].

\section{Conclusion}

Both ATLAS and CMS have extended many searches for new physics with tau leptons. All results have been consistent with the SM background predictions. The reach of these searches will be extended with the $20-30 \mathrm{fb}^{-1}$ of $\sqrt{s}=8 \mathrm{TeV}$ collision data being collected in 2012.

\section{References}

[1] L. R. Evans, P. Bryant, LHC Machine, JINST 3 (2008) S08001.

[2] The ATLAS experiment at the CERN Large Hadron Collider, JINST 3 (2008) S08003.

[3] The CMS experiment at the CERN LHC, JINST 3 (08) (2008) S08004.

[4] ATLAS Collaboration, Performance of the reconstruction and identification of hadronic tau decays with ATLAS, ATLASCONF-2011-152 (2011).

[5] CMS Collaboration, Performance of $\tau$-lepton reconstruction and identification in CMS, JINST 7 (2012) 1001. arXiv:1109.6034.

[6] ATLAS Collaboration, Search for charged Higgs bosons decaying via $H^{+} \rightarrow \tau v$ in top quark pair events using $p p$ collision data at $\sqrt{s}=7 \mathrm{TeV}$ with the ATLAS detector, JHEP 1206 (2012) 039. arXiv: 1204.2760 .

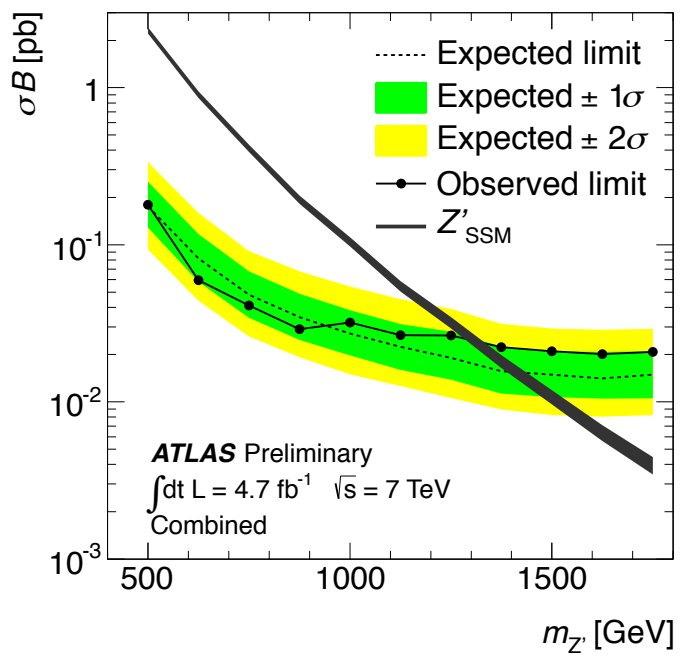

Figure 11: The expected and observed 95\% CL upper limits on the cross section times $\tau^{+} \tau_{\text {had }}^{-}$branching fraction, $\sigma B$, as a function of the $Z^{\prime}$ mass, combining all channels. The combined limit excludes SSM $Z^{\prime}$ masses up to $1.3 \mathrm{TeV}$, with an expected limit of $1.4 \mathrm{TeV}$ [15].

[7] CMS Collaboration, Search for a light charged Higgs boson in top quark decays in pp collisions at $\sqrt{s}=7 \mathrm{TeV}$, JHEP 1207 (2012) 143. arXiv:1205.5736.

[8] CMS Collaboration, Search for new physics with same-sign isolated dilepton events with jets and missing transverse energy, Phys. Rev. Lett. 109 (2012) 071803. arXiv:1205.6615.

[9] CMS Collaboration, Search for new physics in events with opposite-sign leptons, jets, and missing transverse energy in pp collisions at $\sqrt{s}=7 \mathrm{TeV}$. Submitted to Phys. Lett. B. arXiv: 1206.3949

[10] CMS Collaboration, Search for anomalous production of multilepton events in pp collisions at $\sqrt{s}=7 \mathrm{TeV}$, JHEP 1206 (2012) 169. arXiv: 1204.5341

[11] ATLAS Collaboration, Search for supersymmetry in events with large missing transverse momentum, jets, and at least one tau lepton in $7 \mathrm{TeV}$ proton-proton collision data with the ATLAS detector. Submitted to EPJC. arXiv:1210.1314.

[12] CMS Collaboration, Search for pair production of third generation leptoquarks and stops that decay to a tau and a b quark, CMS PAS EXO-12-002 (2012).

[13] CMS Collaboration, Search for narrow resonances in dilepton mass spectra in pp collisions at $\sqrt{s}=7 \mathrm{TeV}$, Phys. Lett. B 714 (2012) 158-179. arXiv: 1206.1849 .

[14] ATLAS Collaboration, Search for high-mass resonances decaying to dilepton final states in pp collisions at a center-of-mass energy of $7 \mathrm{TeV}$ with the ATLAS detector. Submitted to JHEP. arXiv:1209.2535.

[15] ATLAS Collaboration, A search for high-mass resonances decaying to $\tau^{+} \tau^{-}$in pp collisions at $\sqrt{s}=7 \mathrm{TeV}$ with the ATLAS detector, ATLAS-CONF-2012-067 (2012).

[16] ATLAS Collaboration, A search for high-mass resonances decaying to $\tau^{+} \tau^{-}$in $p p$ collisions at $\sqrt{s}=7 \mathrm{TeV}$ with the ATLAS detector. Submitted to Phys. Lett. B. arXiv:1210.6604.

[17] CMS Collaboration, Search for high-mass resonances decaying into $\tau$-lepton pairs in pp collisions at $\sqrt{s}=7 \mathrm{TeV}$, Phys. Lett. B 716 (2012) 82-102. arXiv:1206.1725. 